\title{
Ethical quagmire awaits vaccine for cocaine addiction
}

Treating cocaine addiction has proven a slow and thorny task for scientists. The best bet, a vaccine, is years away, but it has already raised several ethical questions.

There are an estimated 1.5 million cocaine addicts in the US alone and the related costs run to more than $\$ 70$ billion per year. But developing drugs to fight cocaine addiction has proven difficult. Most drugs of abuse, such as heroin, stimulate the brain's pleasure circuit by triggering the release of neurotransmitters. But cocaine steps in at the next stage, blocking the reuptake of the neurotransmitters dopamine and serotonin back into the nerve cells.

"Cocaine is an especially intractable problem," says Donald Landry, an addiction researcher at Columbia University. "It's inherently difficult to block a blocker."

Recruiting the immune system to block cocaine's effects is one alternative. Cocaine molecules are too small to induce an immune response, but by attaching a cocaine-like molecule to a large protein, researchers found they could coax the immune system into making antibodies to both. The antibodies then bind cocaine in the bloodstream, preventing the drug from entering the brain.

Landry and several others are developing immunization strategies, including vaccines and antibodies, for cocaine addiction. But only

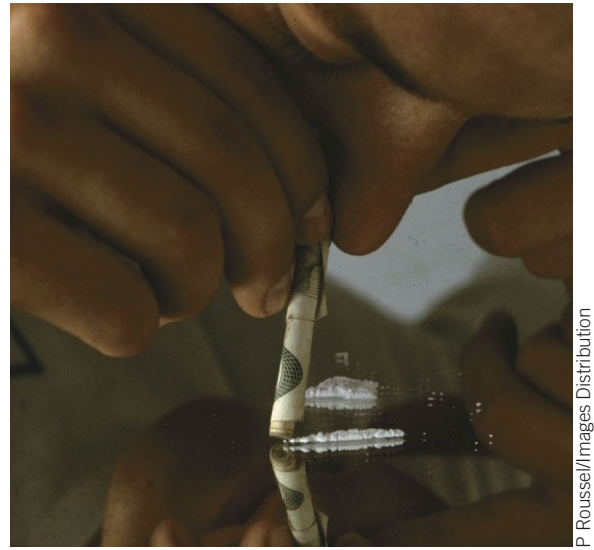

A UK panel has proposed vaccinating peopleincluding children-at high risk of addiction.

one vaccine, licensed to the British biotech company Xenova, is in clinical trials. In Xenova's most recent trial, about $75 \%$ of vaccinated addicts were able to abstain from cocaine for three months, the company reported in August, and subjects who relapsed said the high they felt was less potent than before. The company is set to test the vaccine in a bigger phase 2 trial.

Even an effective vaccine would not arrest cocaine addiction, researchers warn. "The word 'vaccine' is accurate, but perhaps unfortunate," says Paul Pentel, professor of pharmacology at the University of Minnesota. "These are not like vaccines for infectious diseases-it's much more analogous to medication."

What's more, using the vaccine is fraught with ethical pitfalls: can an addict truly consent to treatment? Should governments compel high-risk individuals — such as those convicted for drug use- to be vaccinated? Who would decide who is at risk?

"These issues could really tarnish the potential of a medication that could otherwise be very effective for many people," says Henrick Harwood, who last year chaired an advisory panel convened by the US National Institute on Drug Abuse on the topic.

Creating a framework for discussing those questions in advance could prevent policies that are not rooted in science. In August, a UK government-appointed panel called the Brain Science, Addiction and Drugs Project proposed vaccinating children-potentially even as infants - deemed at risk of using drugs.

Even apart from the ethical dilemmas, such schemes are impractical, researchers say. The vaccine is effective for just three months, requiring four to five doses during that time, notes Landry. "You could immunize all these people," he says, "but you'd be signing them on for monthly injections for the rest of their lives." Alla Katsnelson, New York

\section{Inflamed brains can trigger the blues, studies suggest}

The same chemicals that can induce the body to fight infection can also sicken the brain: a growing body of evidence suggests that inflammation may be linked to depression.

Studies in animals "demonstrate quite convincingly that signals from the immune system get into the brain," says Andrew Miller, professor of psychiatry at Emory University.

Miller says the signaling molecules, or cytokines, can alter the levels of neurotransmitters such as dopamine and serotonin in the brain. In animals, an increase in the level of cytokines can induce 'sickness behavior'-the animal equivalent of depression.

Scientists have known for more than a decade that cytokines such as interferon-alpha and interleukin- 2 can cause depression in cancer patients. Miller's work also suggests that antidepressants given before cytokine therapy can stave off depression. In that study, only $11 \%$ of cancer patients who took the antidepressant paroxetine two weeks before starting cytokine therapy developed depression, compared with $45 \%$ on placebo (N. Engl. J. Med. 344, 961-966; 2001).
More recently, another study showed that treating cancer patients with cytokines lowers blood levels of tryptophan-an essential amino acid that is a precursor of the mood-stabilizing neurotransmitter serotonin. About one-third of patients on the medications became depressed (Mol. Psychiatry 7, 468-473; 2002).

In those patients, the lower the levels of tryptophan, the more severe the depression, says lead investigator Robert Dantzer, director of the Center for Integrative Neurobiology in Bordeaux, France. Dantzer says the cytokines might be activating an enzyme that breaks down tryptophan.

What isn't clear is whether it is byproducts of the enzyme's actions on tryptophan or tryptophan itself that cause the behavioral changes.

In fact, both processes might be responsible. Depression is a complex disorder that appears to involve several separate brain systems. For instance, cytokines can activate the corticotropin-releasing factor pathway, which regulates the stress response. Depression may also involve the dopamine system, which is implicated in addictive behaviors.

"People are starting to talk about different symptom complexes being mediated by different pathways of neurocircuitry," says Miller.

Even if it is established as causing depression, inflammation is unlikely to account for all cases of depression. There is also little research on whether circumstances such as job loss or divorce can exacerbate inflammation.

That the immune system contributes to depression in some cases is becoming “incontrovertible," says Philip Gold, chief of neuroendocrinology at the US National Institute of Mental Health. "But the idea has not been generally accepted because there hasn't been a smoking gun."

Proof of the theory would require reversing brain inflammation with treatment, "but we haven't shown that yet," Gold says. "A system as complex as this is going to yield contradictory data," he adds. "But I really believe [the link] is there. There are too many pieces that are beginning to fit together."

Gunjan Sinha, Frankfurt 